\title{
Estimativa da umidade na capacidade de campo em solos sob Cerrado
}

\author{
Rui da S. Andrade ${ }^{1} \&$ Luís F. Stone $^{2}$
}

\begin{abstract}
RESUMO
O bjetivou-se, com este trabalho, estimar a umidade na capacidade de campo ( $\theta c c)$ a partir de equação baseada na curva de retenção de água do solo (CRA) e de correlações com atributos físico-hídricos e dados de textura e matéria orgânica, para solos muito-argilosos, argilosos, médios e arenosos. 0 estudo foi realizado com 2242 amostras de solo do Cerrado, das quais constavam informações sobre classificação textural, atributos físicos e retenção da água do solo. Para 745 amostras havia ainda informação sobre a condutividade hidráulica saturada (Ko) e, para 472, sobre teores de argila, silte, areia e matéria orgânica. Para as amostras com Ko medida, $\theta c c$ foi determinada com base em parâmetros da equação de G enuchten (1980), assumindo-se que a taxa de drenagem $(\tau)$ é uma porcentagem $p$ de Ko. Consideraram-se valores de $p$ iguais a 0,$0025 ; 0,0050 ; 0,0100 ; 0,0150$ e 0,0200 . Verificou-se, então, ser possível estimar $\theta c c$ a partir de equação baseada na CRA, considerando-se $\tau$ como sendo $1 \%$ do valor de Ko. $\theta c c$ também pode ser estimada em função da umidade no ponto de inflexão da CRA e da porosidade total para cada horizonte do solo. Para $\tau$ equivalente a $1 \%$ do valor de Ko, a tensão de água no solo correspondente à $\theta \mathrm{cc}$ se situou entre 6,5 e 7,5 $\mathrm{kPa}$.
\end{abstract}

Palavras-chave: curva de retenção de água do solo, taxa de drenagem, atributos físicos, textura do solo

\section{Estimation of moisture at field capacity in soils under 'Cerrado'}

\begin{abstract}
The objective of this study was to estimate the soil moisture at field capacity ( $\theta \mathrm{fc}$ ) by an equation based on the soil water retention curve (SWRC) and by correlation with physico-hydrical attributes and data from texture and organic matter for very clayey, clayey, loamy, and sandy soils. The study was carried out with 2242 samples from 'Cerrado' soils, which contained information on textural classification, physical attributes and soil water retention. For 745 samples, there was information on the saturated hydraulic conductivity (Ko) and for 472, on clay, silt, sand and organic matter contents. For samples with measured Ko, $\theta$ fc was determined based on parameters of the equation of Genuchten (1980), assuming that the drainage rate $(\tau)$ is a percentage $p$ of Ko. $p$ values of $0.0025,0.0050,0.0100,0.0150$, and 0.0200 were considered. It was observed that $\theta$ fc can be estimated by the equation based on SWRC, considering $\tau$ as $1 \%$ of the Ko value. $\theta$ fc can also be estimated as a function of moisture at the inflection point of SW RC and soil porosity for each soil horizon. The soil water tension corresponding to $\theta$ fc was between 6.5 and 7.5 $\mathrm{kPa}$, for $\tau$ equivalent to $1 \%$ of the Ko value.
\end{abstract}

Key words: soil water retention curve, drainage rate, physical attributes, soil texture 


\section{INTRODUÇÃO}

O manejo adequado de sistemas de irrigação depende das características físicas e químicas do solo. A interação da água com essas características manifesta propriedades como o limite superior de umidade que determinado solo apresenta, também denominado capacidade de campo, de grande importância nos processos de armazenagem e disponibilidade de água para as plantas. Diversos autores têm discutido as imprecisões do conceito pioneiro de capacidade de campo dado por Veihmeyer $\&$ Hendrickson (1949), em que definem capacidade de campo como "a quantidade de água retida pelo solo depois que o excesso tenha drenado e a taxa de movimento descendente tenha decrescido acentuadamente o que ocorre, geralmente, dois a três dias depois de uma chuva ou irrigação em solos permeáveis de estrutura e textura uniforme". Reichardt (1988) questiona este conceito, quanto à subjetividade, em algumas de suas expressões, excesso de água, tempo ideal de drenagem, solos permeáveis e homogêneos etc., mas o considera importante para os que estiverem interessados apenas no manejo de culturas irrigadas, quando a definição de capacidade de campo se torna útil e prática. Segundo o autor, a capacidade de campo é um processo dinâmico, não uma característica da matriz do solo, que depende de interesses específicos de cada situação. Por exemplo, ele recomenda que, para os irrigantes, a capacidade de campo deve ser determinada depois de dois a três dias pós-chuva ou irrigação, em obediência a um turno de rega menor que dez dias e, para os interessados em lixiviação de nutrientes e pesticidas e recarga de aquíferos subterrâneos, que este tempo seja mais longo.

A capacidade de campo depende do fluxo de drenagem e este por sua vez se acha mais à mercê da condutividade hidráulica do que do potencial total da água no solo. Reduções drásticas do fluxo de água de drenagem foram observadas por Reichardt (1988) em um Latossolo Vermelho-Amarelo de textura média, ao verificar que em apenas um dia esse fluxo reduziu para $0,3 \%$ de seu valor original, devido à redução também significativa da condutividade hidráulica, quando praticamente não houve alteração do gradiente de potencial total de água no solo.

A determinação da tensão que governa a capacidade de campo é, igualmente, motivo de inúmeros trabalhos, sobremaneira daqueles realizados em laboratório, com amostras deformadas e indeformadas. A definição da tensão matricial de água no solo entre $10 \mathrm{kPa}$ e $33 \mathrm{kPa}$ como correspondente à capacidade de campo, tem encontrado resistência entre pesquisadores. Reichardt (1988) notou que a tensão matricial não alcançou o valor clássico de $33 \mathrm{kPa}$, mesmo depois de 45 dias, apesar de reconhecer a não existência da relação direta entre fluxo e tensão da água do solo. Vários autores têm postulado que a capacidade de campo para solos tropicais corresponderia a tensões variando de 6 a $10 \mathrm{kPa}$ (Ferreira \& Marcos, 1983; Reichardt, 1988; Andrade et al., 1991; Mello et al., 2002). A adoção de certa tensão representativa da capacidade de campo é de interesse geral, dado à praticidade de se caracterizar rapidamente a sua umidade correspondente, por meio de curvas características de retenção de água.
Ferreira \& Marcos (1983) propuseram o conceito do ponto de inflexão da curva característica de retenção de água no solo corresponder à capacidade de campo e obtiveram resultados significativos quando este ponto foi correlacionado com a umidade determinada na tensão de $6 \mathrm{kPa}$. Dexter \& Bird (2001), por sua vez, consideraram o ponto de inflexão da curva de retenção de água ajustada pelo modelo de Genuchten (1980) como o ponto ótimo para preparo do solo em termos de umidade, e a capacidade de campo como equivalente à tensão de $10 \mathrm{kPa}$.

Em campo, a determinação da capacidade de campo requer gasto de tempo e tem custos. Sua estimativa, no entanto, por intermédio de modelos matemáticos pode ser uma alternativa econômica, em curto espaço de tempo e de reconhecida viabilidade técnica. As funções de pedotransferência são equações que facilitam a estimativa de características edáficas, de difícil determinação, a partir de outros atributos mais facilmente obtidos (Oliveira et al., 2002). Macedo (1991) desenvolveu funções de pedotransferência a partir de uma classificação textural, teor de matéria orgânica e microporosidade (tensão igual a $6 \mathrm{kPa}$ ), para estimar a capacidade de campo à base de volume.

Objetivou-se com este trabalho verificar a possibilidade de se estimar a umidade na capacidade de campo a partir de equação baseada na curva de retenção de água do solo e de correlações com atributos físico-hídricos e dados de textura e matéria orgânica, para solos muito-argilosos, argilosos, médios e arenosos.

\section{Material e MÉTODOS}

O estudo foi realizado com 2242 amostras de solo, das quais constavam informações sobre classificação textural, densidade do solo (Ds) e de partículas (Dp), e retenção da água do solo, registradas nos bancos de dados dos Laboratórios de Solo da Embrapa Arroz e Feijão e Embrapa Cerrados. Para 745 amostras havia ainda informação sobre a condutividade hidráulica saturada (Ko) e para 472 sobre teores de argila, silte, areia e matéria orgânica. Essas amostras abrangiam solos sob Cerrado das regiões Centro-Oeste, Norte e Nordeste do Brasil.

Os dados de retenção da água do solo foram ajustados a uma curva pela equação de Genuchten (1980), que é dada por:

$$
\theta=(\theta \mathrm{s}-\theta \mathrm{r})\left[1+(\alpha \mathrm{h})^{\mathrm{n}}\right]^{-\mathrm{m}}+\theta \mathrm{r}
$$

em que $\theta, \theta$ s e $\theta$ r são, respectivamente, os conteúdos de água do solo correspondentes à tensão $\mathrm{h}$, à saturação e à umidade residual, $\mathrm{em} \mathrm{m}^{3} \mathrm{~m}^{-3}$, h é a tensão matricial da água do solo, em $\mathrm{kPa}, \mathrm{n}$ e $\mathrm{m}(\mathrm{m}=1-1 / \mathrm{n})$ são parâmetros empíricos adimensionais de ajuste e $\alpha$ é um parâmetro expresso em $\mathrm{kPa}^{-1}$. O ajuste foi feito com o auxílio do programa Soil Water Retention Curve SWRC (Dourado Neto et al., 2001).

Para as 745 amostras com Ko medida, a umidade na capacidade de campo $(\theta \mathrm{cc})$ foi determinada com base nas equações para determinação da densidade de fluxo 
gravitacional e da umidade do solo na profundidade de interesse L, de acordo com o tempo de redistribuição da água do solo, propostas por Loyola \& Prevedello (2003), que utilizam parâmetros da equação de Genuchten (1980).

O tempo correspondente à capacidade de campo (Tcc), em $\mathrm{h}$, pode ser expresso como:

$$
\mathrm{Tcc}=\frac{\mathrm{L}(\theta \mathrm{s}-\theta \mathrm{r})}{(1-\gamma) \mathrm{Ko}}\left(1-\left(\frac{\tau}{\mathrm{Ko}}\right)^{\frac{1-\gamma}{\gamma}}\right)
$$

em que

$$
\tau=\left|-\frac{\mathrm{d} \theta}{\mathrm{dt}} \mathrm{L}\right|
$$

é a densidade de fluxo gravitacional ou taxa de drenagem, agora com a conotação de fluxo no momento em que o solo está em seu limite superior, ou seja, na capacidade de campo,

$$
\gamma=2,5+\frac{2}{n-1}
$$

\section{e Ko é expressa em $\mathrm{cm} \mathrm{h}^{-1}$.}

Substituindo-se a equação (2) na equação proposta por Loyola \& Prevedello (2003) para determinação da umidade do solo na profundidade de interesse, tem-se:

$$
\theta \mathrm{cc}=(\theta \mathrm{s}-\theta \mathrm{r})\left(1-\left(1-\left(\frac{\tau}{\mathrm{Ko}}\right)^{\frac{1-\gamma}{\gamma}}\right)\right)^{\frac{1}{1-\gamma}}+\theta \mathrm{r}
$$

Assumindo que a condição de capacidade de campo é atingida quando a taxa de drenagem $\tau$ se reduz para dada porcentagem $\mathrm{p}$ da condutividade hidráulica saturada, conforme proposto por Prevedello (1999), a Equação 3 se simplifica para:

$$
\theta \mathrm{cc}=(\theta \mathrm{s}-\theta \mathrm{r})\left(1-\left(1-\mathrm{p}^{\frac{1-\gamma}{\gamma}}\right)\right)^{\frac{1}{1-\gamma}}+\theta \mathrm{r}
$$

A equação para obtenção da tensão correspondente à capacidade de campo (hcc) é dada por:

$$
\mathrm{hcc}=\frac{\left(\left(\frac{\theta \mathrm{s}-\theta \mathrm{r}}{\theta \mathrm{cc}-\theta \mathrm{r}}\right)^{\frac{1}{\mathrm{~m}}}\right)^{1 / \mathrm{n}}}{\alpha}
$$

O tempo correspondente à capacidade de campo (Tcc) agora pode ser calculado por:

$$
\mathrm{Tcc}=\frac{\mathrm{L}(\theta \mathrm{s}-\theta \mathrm{r})}{(1-\gamma) \mathrm{Ko}}\left(1-(\mathrm{p})^{\frac{1-\gamma}{\gamma}}\right)
$$

Considerou-se Ligual a $20 \mathrm{~cm}$ e o Tcc foi determinado para valores de p iguais a 0,$0025 ; 0,0050 ; 0,0100 ; 0,0150$ e 0,0200 . Os valores obtidos foram comparados com os da literatura, para determinar o valor mais adequado de p. Para este valor foi calculada $\theta \mathrm{cc}$ e hcc para todas as 2242 amostras, separadas por classe textural muito argilosa (teor de argila (Ag) maior ou igual à $60 \%)$, argilosa $(60 \%>\mathrm{Ag}$ maior ou igual à 35\%), média $(35 \%>\mathrm{Ag}$ maior ou igual à $15 \%)$ e arenosa $(\mathrm{Ag}<15 \%)$.

Considerando-se todas as amostras estabeleceram-se correlações múltiplas entre a $\theta \mathrm{cc}$, determinada com o valor estabelecido de $\mathrm{p}$, e os atributos densidade do solo, em $\mathrm{Mg} \mathrm{m}^{-3}$, porosidade total, macroporosidade, microporosidade e umidade do solo no ponto de inflexão da curva de retenção da água, todas expressas em $\mathrm{m}^{3} \mathrm{~m}^{-3} \mathrm{e}$ índice $\mathrm{S}$.

Calculou-se a porosidade total $(\mathrm{Pt})$ pela equação: $\mathrm{Pt}=(1-$ Ds/Dp). A microporosidade foi considerada a quantidade de água retida pelo solo na tensão de $6 \mathrm{kPa}$. Obteve-se a macroporosidade pela diferença entre a porosidade total e a microporosidade (EMBRAPA, 1997).

Determinou-se a umidade no ponto de inflexão da curva de retenção da água do solo $(\theta \mathrm{i})$ de acordo com Dexter \& Bird (2001) por:

$$
\theta \mathrm{i}=(\theta \mathrm{s}-\theta \mathrm{r})\left[1+\frac{1}{\mathrm{~m}}\right]^{-\mathrm{m}}+\theta \mathrm{r}
$$

O índice $S$, que é a declividade da curva de retenção da água do solo em seu ponto de inflexão, foi calculado segundo Dexter (2004) por:

$$
\mathrm{S}=-\mathrm{n}(\theta \mathrm{s}-\theta \mathrm{r})\left[1+\frac{1}{\mathrm{~m}}\right]^{-(1+\mathrm{m})}
$$

Estabeleceram-se, também, correlações múltiplas entre a umidade na capacidade de campo expressa à base de massa, determinada com o valor estabelecido de p, e os teores de argila, silte, areia e matéria orgânica, para as 472 amostras de que se tinham essas informações.

\section{RESULTADOS E DISCUSSÃO}

Considerando-se as 745 amostras com dados de Ko, os valores de p iguais a 0,0100 e 0,0150 propiciaram valores de Tcc entre 63,1 e 44,1 h (Tabela 1), muito próximos da recomendação de Reichardt (1988) para profissionais em irrigação, de que a capacidade de campo seja determinada dois a três dias após o processo de molhamento do solo, quando o turno de rega for menor que dez dias. 
Tabela 1. Tempo médio para atingir a capacidade de campo (Tcc), em razão de valores da relação entre a taxa de drenagem e a condutividade hidráulica saturada (p)

\begin{tabular}{cr}
\hline Valor de $\mathbf{p}$ & Tcc (h) \\
0,0025 & 213,4 \\
0,0050 & 116,3 \\
0,0100 & 63,1 \\
0,0150 & 44,1 \\
0,0200 & 34,1 \\
\hline
\end{tabular}

Desta forma se consideraram, de início, os valores de $\mathrm{p}$ iguais a 0,0100 e 0,0150 no cálculo de $\theta$ cc e hcc com a utilização da equação 4, para todas as 2242 amostras, separadas por classe textural. Observa-se que o uso desses valores de p resultou em valores de hcc entre 5,7 e 7,5 kPa, dependendo da textura do solo (Tabela 2).

Tabela 2. Valores da umidade na capacidade de campo $(\theta c c)$ e da tensão correspondente (hcc), em razão de valores da relação entre a taxa de drenagem e a condutividade hidráulica saturada $(p)$

\begin{tabular}{lccccc}
\hline \multirow{2}{*}{$\begin{array}{c}\text { Classe } \\
\text { textural }\end{array}$} & \multicolumn{2}{c}{$\mathbf{p = 0 , 0 1 0 0}$} & & \multicolumn{2}{c}{$\mathbf{p = 0 , 0 1 5 0}$} \\
\cline { 2 - 3 } \cline { 5 - 6 } & $\begin{array}{c}\theta \mathbf{c c} \\
\left(\mathbf{m}^{\mathbf{3}} \mathbf{~ m}^{-3}\right)\end{array}$ & $\begin{array}{c}\mathbf{h c c} \\
(\mathbf{k P a})\end{array}$ & & $\begin{array}{c}\theta \mathbf{~ c c} \\
\left(\mathbf{m}^{\mathbf{3}} \mathbf{~ m}^{-3}\right)\end{array}$ & $\begin{array}{c}\mathbf{h c c} \\
(\mathbf{k P a})\end{array}$ \\
Muito argilosa & 0,409 & 7,5 & & 0,417 & 6,5 \\
Argilosa & 0,401 & 7,4 & & 0,409 & 6,4 \\
Média & 0,354 & 6,8 & & 0,362 & 6,0 \\
Arenosa & 0,239 & 6,5 & & 0,249 & 5,7 \\
\hline
\end{tabular}

Esses valores estão próximos do valor $6 \mathrm{kPa}$, que pode ser considerado alternativa viável para estimação da umidade na capacidade de campo, quando se tem a curva característica de retenção de água dos solos (Ferreira \& Marcos, 1983; Andrade et al., 1991). Mello et al. (2002) verificaram que o limite superior da água disponível correspondeu a $6 \mathrm{kPa}$, sendo estatisticamente igual ao ponto de inflexão da curva característica de retenção, quando o modelo de ajuste da curva de retenção de água do solo se tratar de uma polinomial cúbica.

Em um solo de textura argilosa, Dardengo et al. (2005) determinaram o valor de $0,396 \mathrm{~m}^{3} \mathrm{~m}^{-3}$ para a capacidade de campo, pelo método direto em vasos, valor bem próximo da média de $0,401 \mathrm{~m}^{3} \mathrm{~m}^{-3}$ obtida com $\mathrm{p}=0,0100$ (Tabela 2 ). Souza et al. (2000) obtiveram, em solo de textura média e pelo método direto em casa de vegetação, o valor de $0,347 \mathrm{~m}^{3} \mathrm{~m}^{-3}$, valor próximo à média $0,354 \mathrm{~m}^{3} \mathrm{~m}^{-3}$ informada nesta tabela para $\mathrm{o}$ mesmo valor de p. Em solo de textura arenosa, Fabian \& Ottoni
Filho (2000) encontraram, na determinação in situ, o valor médio de $0,210 \mathrm{~m}^{3} \mathrm{~m}^{-3}$ no perfil de $0,60 \mathrm{~m}$, próximo ao valor médio obtido com p =0,0100; já Souza et al. (2002) determinaram a capacidade de campo de um solo arenoso pelo método direto em casa de vegetação e encontraram o valor de $0,254 \mathrm{~m}^{3} \mathrm{~m}^{-3}$, mais próximo da média de capacidade de campo para $\mathrm{p}=0,0150$, que foi $0,249 \mathrm{~m}^{3} \mathrm{~m}^{-3}$ (Tabela 2).

Apresenta-se, na Tabela 3, a comparação entre umidades na capacidade de campo medidas por diferentes metodologias e as obtidas com a aplicação da equação 4 , para $\mathrm{p}=0,0100$ e $p=0,0150$. A correlação entre $\theta$ cc medida e a estimada com $\mathrm{p}=0,0100$ foi expressa pela equação $\theta \mathrm{cc}$ estimada $=1,090$ $\theta$ cc medida, $\mathrm{R}^{2}=0,83^{* *}$. Para $\mathrm{p}=0,0150$, a equação foi Өcc estimada $=1,125$ qcc medida, $\mathrm{R}^{2}=0,83 * *$. Observou-se tendência dos valores de capacidade de campo estimados se aproximarem mais dos valores medidos quando se utilizou $\mathrm{p}=0,0100$.

Prevedello (1999) verificou, para uma Terra Roxa Estruturada, que os valores de umidade ou tempo na capacidade de campo pouco diferiram para $p=0,0150$ ou 0,0100 . Com base neste resultado e no obtido para um Latossolo Vermelho-Amarelo fase arenosa, o autor concluiu que a adoção de $\tau$ como sendo $1 \%$ do valor Ko, tanto para o solo homogêneo quanto para o heterogêneo, foi capaz de estimar a umidade na capacidade de campo com valores bem próximos daqueles observados em condições de campo. A comparação entre os resultados obtidos no presente trabalho e os dados da literatura, corrobora com esta assertiva.

Considerando-se todas as 2242 amostras, as correlações entre $\theta \mathrm{cc}$ determinada pela equação 4 para $\mathrm{p}=0,0100$, e os atributos físico-hídricos que propiciaram os mais altos valores de $\mathrm{R}^{2}$, foram:
a) Para uma variável independente
$\theta c c=1,0167 \theta \mathrm{i}-0,0418, \mathrm{R}^{2}=0,92 * *$
b) Para duas variáveis independentes
$\theta \mathrm{cc}=1,5663 \theta \mathrm{i}-0,5207 \mathrm{Pt}-0,0063, \mathrm{R}^{2}=0,98 * *$
c) Para três variáveis independentes
$\theta c c=1,4345 \theta \mathrm{i}-0,4038 \mathrm{Pt}-0,1365 \mathrm{~S}-0,0036, \mathrm{R}^{2}=0,99 * *$
d) Para quatro variáveis independentes
$\theta c c=1,4476 \theta \mathrm{i}-0,4129 \mathrm{Pt}-0,1498 \mathrm{~S}-0,0099 \mathrm{Ds}+0,0095, \mathrm{R}^{2}=$ $0,99 * *$

Verifica-se que, quando se considerou apenas uma variável independente, a melhor correlação foi com $\theta \mathrm{i}$, o que corrobora com a proposição de Ferreira \& Marcos (1983) e Mello et al. (2002) de considerarem a umidade no ponto de inflexão da curva

Tabela 3. Valores da umidade na capacidade de campo $(\theta c c)$ medida e determinada pela equação 4 para diferentes valores da relação entre a taxa de drenagem e a condutividade hidráulica saturada ( $p$ )

\begin{tabular}{|c|c|c|c|c|c|}
\hline \multirow{2}{*}{ Classe textural } & \multirow{2}{*}{ Referência } & \multirow{2}{*}{ Método } & \multirow{2}{*}{$\theta \mathrm{cc}\left(\mathrm{m}^{3} \mathrm{~m}^{-3}\right)$ medida } & \multicolumn{2}{|c|}{$\theta \mathrm{cc}\left(\mathrm{m}^{3} \mathrm{~m}^{-3}\right)$} \\
\hline & & & & $p=0,0100$ & $p=0,0150$ \\
\hline Muito argilosa & Polyanna et al. (2005) & $\mathrm{PI}$ & 0,321 & 0,348 & 0,356 \\
\hline Muito argilosa & Casaroli \& van Lier (2008) & Vaso & 0,322 & 0,384 & 0,395 \\
\hline Argilosa & Polyanna et al. (2005) & PI & 0,334 & 0,357 & 0,364 \\
\hline Argilosa & Polyanna et al. (2005) & $\mathrm{PI}$ & 0,325 & 0,372 & 0,379 \\
\hline Argilosa & Costa et al. (1997) & Tubo & 0,323 & 0,323 & 0,337 \\
\hline Média & Costa et al. (1997) & Tubo & 0,250 & 0,276 & 0,293 \\
\hline Média & Casaroli \& van Lier (2008) & Vaso & 0,243 & 0,245 & 0,257 \\
\hline
\end{tabular}


de retenção de água no solo como a capacidade de campo. Fabian \& Ottoni Filho (2000), testando equações com uma, três e cinco variáveis independentes para estimar a capacidade de campo de um Podzólico Vermelho-Amarelo, constataram que o menor erro relativo foi obtido com a equação que considerava apenas uma variável, a microporosidade (mp). No presente trabalho e apesar da correlação entre $\theta \mathrm{cc}$ e mp ser altamente significativa $\left(\theta \mathrm{cc}=0,7519 \mathrm{mp}+0,098, \mathrm{R}^{2}=0,57 * *\right)$, o coeficiente de determinação foi menor do que quando se consideraram outros atributos, significando que considerar a umidade na tensão de $6 \mathrm{kPa}$ como igual à capacidade de campo, pode não corresponder à realidade. Observou-se, ainda, que o uso de duas variáveis independentes, $\theta$ i e Pt, foi suficiente para explicar a quase totalidade da variabilidade de $\theta$ cc.

As correlações entre a umidade na capacidade de campo determinada pela equação 4 para $p=0,0100$ e expressa à base de massa (Ucc), e os teores de argila (Ag), silte (Silt), areia (Ar) e matéria orgânica (MO) que propiciaram os mais altos valores de $\mathrm{R}^{2}$, considerando-se as 472 amostras de solo, foram:

a) Para uma variável independente
$\mathrm{Ucc}=0,0023 \mathrm{Ag}+0,2192, \mathrm{R}^{2}=0,61 * *$

b) Para duas variáveis independentes

$\mathrm{Ucc}=0,0019 \mathrm{Ag}+0,0024 \mathrm{Silt}+0,2143, \mathrm{R}^{2}=0,70 * *$

$\mathrm{Ucc}=0,0020 \mathrm{Ag}+0,0145 \mathrm{MO}+0,2025, \mathrm{R}^{2}=0,70 * *$

c) Para três variáveis independentes

$\mathrm{Ucc}=0,0018 \mathrm{Ag}+0,0019 \mathrm{Silt}+0,0111 \mathrm{MO}+0,2026$, $\mathrm{R}^{2}=0,75^{* *}$

d) Para quatro variáveis independentes

$\mathrm{Ucc}=0,0026 \mathrm{Ag}+0,0025 \mathrm{Silt}+0,0011 \mathrm{Ar}+0,0108 \mathrm{MO}+0,1253$, $\mathrm{R}^{2}=0,77 * *$

Silva et al. (2008) verificaram forte influência da argila na retenção de água de um Latossolo Vermelho, sendo o único componente textural usado para ajustar funções de pedotransferência. Giarola et al. (2002) encontraram o valor de $\mathrm{R}^{2}=0,79$ para a correlação entre a umidade na capacidade de campo e os teores de argila e de silte de solos da região Sul do Brasil.

Verifica-se que o uso de três variáveis independentes, Ag, Silt e MO, já explica suficientemente a variabilidade de Ucc, não sendo relevante a adição do teor de areia na equação de regressão. Como os valores do coeficiente de determinação não foram muito altos, recomenda-se cautela no uso dessas equações de pedotransferência.

\section{CONClusõEs}

1. É possível estimar a umidade na capacidade de campo a partir de equação baseada na curva de retenção de água do solo, considerando-se a taxa de drenagem como sendo $1 \%$ do valor da condutividade hidráulica saturada em cada horizonte do perfil do solo ou em solos homogêneos.

2. A umidade na capacidade de campo também pode ser estimada em função da umidade no ponto de inflexão da curva de retenção de água e da porosidade total para cada horizonte do solo.
3. Para uma taxa de drenagem equivalente a $1 \%$ do valor da condutividade hidráulica saturada do solo, a tensão de água no solo correspondente à umidade na capacidade de campo se situou entre 6,5 e 7,5 kPa.

\section{AgRAdeCIMENTOS}

Os autores agradecem ao pesquisador da Embrapa Cerrados, Dr. Euzébio Medrado da Silva, pelas informações fornecidas sobre análises fisico-hídricas de amostras de solo armazenadas no banco de dados dessa instituição.

\section{LITERATURA CITADA}

Andrade, C. L. T.; Freitas, J. A. D.; Luz, L. R. Q. P. Características físico-hídricas de solos arenosos de tabuleiro litorâneos. In: Congresso Nacional de Irrigação e Drenagem, 9, 1991, Natal. Anais... Natal: ABID, 1991. v.1, p.1069-1095.

Casaroli, D.; van Lier, Q. J. Critérios para determinação da capacidade de vaso. Revista Brasileira de Ciência do Solo, v.32, p.59-66, 2008.

Costa, A. C. S.; Nani, M. R.; Jeske, E. Determinação da umidade na capacidade de campo e ponto de murchamento permanente por diferentes metodologias. Revista Unimar, v.19, p.827-844, 1997.

Dardengo, M. C. J. D.; Effgen, T. A. M.; Tatagiba, S. D.; Reis, E. F.; Passos, R. R. Avaliação da capacidade de campo em dois solos da região sul do estado do Espírito Santo por diferentes métodos de determinação. In: Encontro LatinoAmericano de Iniciação Científica, 8.; Encontro LatinoAmericano de Pós-Graduação, 5, 2005, São José dos Campos. Anais... São José dos Campos: UNIVAP, 2005. p.1736-1739.

Dexter, A. R. Soil physical quality. Part I. Theory, effects of soil texture, density, and organic matter, and effects on root growth. Geoderma, v.120, p.201-214, 2004.

Dexter, A. R.; Bird, N. R. A. Methods for predicting the optimum and the range of soil water contents for tillage based on the water retention curve. Soil \& Tillage Research, v.57, p.203$212,2001$.

Dourado Neto, D.; Nielsen, D. R.; Hopmans, J. W.; Reichardt, K.; Bacchi, O. O. S.; Lopes, P. P. Programa para confecção da curva de retenção de água no solo, modelo van Genuchten. Soil Water Retention Curve, SWRC (version 3,00 beta). Piracicaba: Universidade de São Paulo, 2001.

EMBRAPA - Empresa Brasileira de Pesquisa Agropecuária. Centro Nacional de Pesquisa de Solos. Manual de métodos de análise de solos. 2.ed. Rio de Janeiro: Embrapa CNPS, 1997. 212p. Documentos, 1.

Fabian, A. J.; Ottoni Filho, T. B. Determinação de capacidade de campo in situ ou através de equações de regressão. Pesquisa Agropecuária Brasileira, v.35, p.1029-1036, 2000.

Ferreira, M. M.; Marcos, Z. Z. Estimativa da capacidade de campo de Latossolo Roxo distrófico e regossolo através do ponto de inflexão da curva característica de umidade. Ciência e Prática, v.7, p.96-101, 1983. 
Genuchten, M. Th. van. A closed-form equation for predicting the hydraulic conductivity of unsaturated soils. Soil Science Society of America Journal, v.44, p.892-898, 1980.

Giarola, N. F. B.; Silva, A. P.; Imhoff, S. Relações entre propriedades físicas e características de solos da região Sul do Brasil. Revista Brasileira de Ciência do Solo, v.26, p.885893, 2002.

Loyola, J. M. T.; Prevedello, C. L. Modelos analíticos para predição do processo da redistribuição da água no solo. Revista Brasileira de Ciência do Solo, v.27, p.783-787, 2003.

Macedo, J. R. Determinação de retenção hídrica por correlação múltipla e de variabilidade espacial em solos podzólicos de Seropédica, RJ. Rio de Janeiro: UFRRJ, 1991. 144p. Dissertação Mestrado

Mello, C. R.; Oliveira, G. C.; Resck, D. V. S.; Lima, J. M.; Dias Júnior, M. S. Estimativa da capacidade de campo baseada no ponto de inflexão da curva característica. Ciência e Agrotecnologia, v.26, p.835-841, 2002.

Oliveira, L. B.; Ribeiro, M. R.; Jacomine P. K. T.; Rodrigues, J. J. V.; Marques, F. A. Funções de pedotransferência para predição da umidade retida a potenciais específicos em solos do estado de Pernambuco. Revista Brasileira de Ciência do Solo, v.26, p.315-323, 2002.
Polyanna, M. O.; Silva, A. M. da; Coelho, C.; Silva, R. A. da. Análise comparativa da caracterização físico-hídrica de um Latossolo Vermelho distrófico in situ e em laboratório. Irriga, v.10, p.1-19, 2005.

Prevedello, C. L. Novo método para estimativa da umidade do solo na condição de capacidade de campo. Revista Brasileira de Recursos Hídricos, v.4, p.23-28, 1999.

Reichardt, K. Capacidade de campo. Revista Brasileira de Ciência do Solo, v.12, p.211-216, 1988.

Silva, A. P. da; Tormena, C. A.; Fidalski, J.; Imhoff, S. Funções de pedotransferência para as curvas de retenção de água e de resistência do solo à penetração. Revista Brasileira de Ciência do Solo, v.32, p.1-10, 2008.

Souza, C. C.; Oliveira, F. A.; Silva, I. F.; Amorim Neto, M. S. Avaliação de métodos de determinação de água disponível e manejo da irrigação em terra roxa sob cultivo de algodoeiro herbáceo. Revista Brasileira de Engenharia Agrícola e Ambiental, v.4, p.338-342, 2000.

Souza, C. C.; Oliveira, F. A.; Silva, I. F.; Amorim Neto, M. S. Avaliação de métodos de determinação de água disponível em solo cultivado com algodão. Pesquisa Agropecuária Brasileira, v.37, p.337-341, 2002.

Veihmeyer, F. J.; Hendrickson, H. Methods of measuring field capacity and permanent wilting percentages of soils. Science, v.68, p.75-94, 1949. 